\title{
Output Power and Efficiency of Electromagnetic Energy Harvesting Systems with Constrained Range of Motion
}

\author{
M. Hendijanizadeh ${ }^{1}$, S. M Sharkh ${ }^{1}$, S. J. Elliott ${ }^{2}$, M. Moshrefi-Torbati ${ }^{1}$ \\ ${ }^{1}$ Electro-Mechanical Engineering Research Group, Engineering Science, \\ Southampton, SO17 1BJ, UK \\ ${ }^{2}$ ISVR, University of Southampton, Southampton, SO17 1BJ, UK \\ E-mail: m.hendijanizadeh@soton.ac.uk
}

\begin{abstract}
In some energy harvesting systems, the maximum displacement of the seismic mass is limited due to the physical constraints of the device. This is especially the case where energy is harvested from a vibration source with large oscillation amplitude (e.g., marine environment). For the design of inertial systems, the maximum permissible displacement of the mass is a limiting condition. In this paper the maximum output power and the corresponding efficiency of linear and rotational electromagnetic energy harvesting systems with a constrained range of motion are investigated. A unified form of output power and efficiency is presented to compare the performance of constrained linear and rotational systems. It is found that rotational energy harvesting systems have a greater capability in transferring energy to the load resistance than linear directly coupled systems, due to the presence of an extra design variable viz. the ball screw lead. Also, in this paper it is shown that for a defined environmental condition and a given proof mass with constrained throw, the amount of power delivered to the electrical load by a rotational system can be higher than the amount delivered by a linear system. The criterion that guarantees this favorable design has been obtained.
\end{abstract}

Keywords- Constrained systems; Efficiency; Energy harvesting; Scaling effect; Vibration,

\section{Introduction}

Generating electricity from ambient vibration has been the subject of significant research and development in the last decade. Piezoelectric [1,2] ,electrostatic [3] and electromagnetic [2, 4-8] transducers are the most commonly used methods that are utilized to convert ambient vibrations to the electrical energy. However, due to the limitation of geometry and limited permissible deformation of electrostatic and piezoelectric transducers, the electromagnetic induction method is the more appropriate choice for large scale applications [9]. Movement of a backpack carried by a human during walking [10], all-terrain-vehicle vibration [11], vertical movement of a sailing boat [12] are some examples of relatively large scale vibration resource.

However, in addition to the configuration of energy conversion device, maximizing the output power and efficiency are the main concerns in the process of design and optimization of vibration energy harvesters. Efficiency is a fundamental parameter used to compare all kinds of energy harvesters with various sizes and designs [13-15]. Usually the main goal of an energy harvesting system is to extract the maximum power from the environment. In this paper, the efficiency of such systems when achieving maximum power is studied. To achieve the maximum power condition, the parameters of the system need to be selected and tuned carefully. Tuning the load resistance to its optimum value to ascertain impedance matching in electromagnetic energy harvesters is reported in many research works to have improved energy capture [16-18]. However, none of these works considered the maximum allowable displacement of the oscillating mass as a constraint in the process of calculating the optimum load resistance. More specifically, the optimum load resistance for harvesting maximum amount of energy is generally calculated regardless of its effect on the relative displacement of the oscillating mass. However, it is known that the load resistance can influence the overall system damping and hence the relative displacement of the mass. In many transducers that are used in large size applications, due to size limitations, the oscillating mass only moves within a specified range. Now, if the load resistance of the transducer is selected without considering the maximum permissible through of the seismic mass, there is a risk that the amplitude of the oscillating mass will exceed the physical dimensions of the transducer 
thus affecting the performance of the device. Therefore, for these cases, at the design stage, the physical parameters such as load resistance should be selected with regard to the constraints on the oscillating mass.

In this paper, the maximum output power and the corresponding efficiency of two types of electromagnetic energy harvesting systems (i.e. linear and rotational) for those with constraints on their range of motion are studied. In linear electromagnetic energy harvesting systems (henceforth referred to as linear system) such as those studied in [11, 19-21] a linear generator is employed. However, in a rotational energy harvesting system (henceforth referred to as rotational system), an intermediate mechanism, such as rack and pinion[7, 10, 22] or a ball screw[23-26], is utilized to convert linear motion of the mass to rotational one to drive a rotary generator.

The paper is distinguished by three main contributions. First it investigates the optimum load resistance for both constrained linear and rotational systems to address the maximum output power condition. It is shown that for constrained systems the optimal load resistance is different from that of unconstrained energy harvesting systems that is reported in literature [16]. Then the efficiency of both systems corresponding to their maximum output power is obtained. For each system, an expression for the load resistance corresponding to maximum efficiency is also derived. It is shown that for linear systems it is not possible to achieve maximum efficiency when the maximum power is extracted from the transducer. However, for rotational systems maximum efficiency occurs at maximum output power point.

The second contribution is the derivation of equations for power and corresponding efficiency of both systems in unified forms so that proper comparison between them can be made. These unified forms are developed based on the non-dimensional electromechanical coupling coefficient of systems introduced by Elliott and Zilletti [27]. The comparison reveals that in the case of a linear system, the maximum amount of power that can be transferred to the load is half the mechanical power transferred by the harvester and the efficiency of system is always less than $50 \%$. However, a rotational system can be designed so as to have an efficiency greater than $50 \%$. The criterion that guarantees the efficiency of a rotational system is more than $50 \%$ is derived.

The third contribution is studying the effect of scaling the size of electromagnetic generator component of the energy harvesting system on the output power and efficiency. It is shown that by increasing the size of energy harvesting system the efficiency is increased for both constrained linear and rotational systems.

\section{Power generation from a constrained inertia system}

\subsection{Power Generation from a constrained linear energy harvesting transducer}

A schematic diagram of a linear energy harvesting system using an electromagnetic generator is shown in figure 1. In this diagram, $m$ is the seismic mass, $k$ is the spring stiffness, $c_{m}$ represents the mechanical viscous damping coefficient, and $c_{e}$ is the electrical damping coefficient corresponding to the combined power dissipated in the generator's internal resistance and the power delivered to the load.

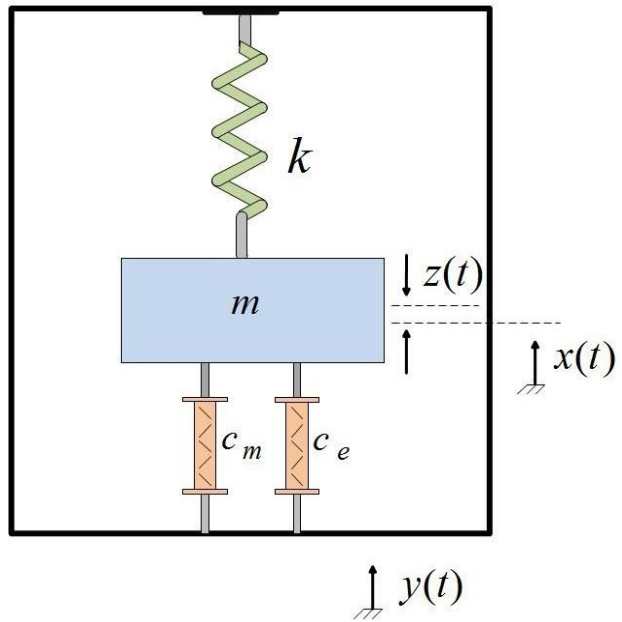

Figure 1. Schematic diagram of a linear energy harvesting system 
The governing differential equation of motion for the system shown in figure 1, with respect to the relative displacement of the seismic mass $z=x-y$, is

$$
m \ddot{z}+\left(c_{e}+c_{m}\right) \dot{z}+k z=-m \ddot{y} .
$$

For a harmonic base excitation $y=Y \sin \left(\omega t+\varphi_{y}\right)$, when the driving motion is assumed to be independent of the mechanical loading due to the harvester, the amplitude of the relative displacement $Z$, can be shown to be

$$
\left|\frac{Z}{Y}\right|=\frac{m \omega^{2}}{\left[\left(k-m \omega^{2}\right)^{2}+\left(\left(c_{e}+c_{m}\right) \omega\right)^{2}\right]^{1 / 2}} .
$$

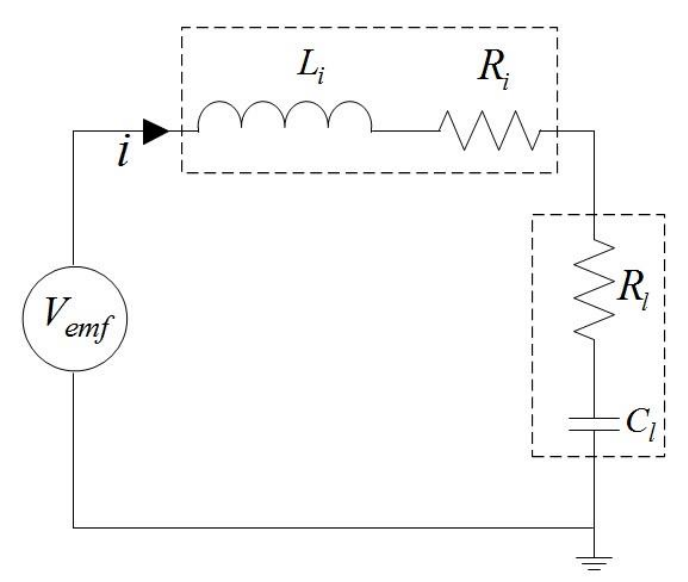

Figure 2. Equivalent circuit of an electromagnetic generator connected to a resistive load.

In many papers on generating energy from vibrations, the effect of the generator's internal inductance is ignored. Cammarano et al. [17] show that even in cases where the effect of internal inductance cannot be ignored, due to a high oscillation frequency, the undesirable effect of the internal resistance can be compensated by adding a capacitor in series with the circuit. The equivalent electrical circuit of the energy harvesting device is shown in figure 2, in which a capacitor is added in series with the load reactance to cancel the effect of the generator's inductance. Assuming that an electrical generator with an emf constant $K_{t}$, is directly coupled to the seismic mass, then the generated emf voltage is given by

$$
V_{\text {emf }}=K_{t} \dot{z}
$$

Also, the electrical damping coefficient $\left(c_{e}\right)$, corresponding to the power dissipated in the generator's internal resistance and transferred to the electrical load, is

where

$$
c_{e}=\frac{K_{t}^{2}}{\left|Z_{l}+Z_{i}\right|}
$$

$$
Z_{i}=R_{i}+\chi_{i}
$$

and

$$
Z_{l}=R_{l}+\chi_{l},
$$

where $\chi_{i}=j L_{i} \omega$ and $\chi_{l}=1 / j C_{l} \omega$. In [17] it is shown that to deliver the maximum power to the load $R_{l}$, the effect of internal inductance should be compensated by tuning the capacitor such that $\chi_{l}=-\chi_{i}$.

For a spring stiffness of $k$, the natural frequency of system is equal to the base excitation frequency when $k=m \omega^{2}$ at which the corresponding relative displacement $Z_{r}$ can be derived from (2) (for $\omega=\omega_{n}$ ). 
Then $z=Z_{r} \sin \left(\omega_{n} t+\varphi_{z}\right)$ and $\dot{z}=Z_{r} \omega_{n} \cos \left(\omega_{n} t+\varphi_{z}\right)$, by substituting the electrical damping coefficients from (4) and considering $C_{l}=1 / L_{i} \omega_{n}^{2}$, the amplitude of the relative displacement is

$$
\frac{Z_{r}}{Y}=\frac{m \omega_{n}}{c_{m}+\frac{K_{t}^{2}}{R_{i}+R_{l}}}
$$

The power delivered to the load resistance is

$$
P_{l-o u t}=\frac{1}{2} R_{l} i^{2}=\frac{1}{2} R_{l}\left(\frac{V_{e m f}}{R_{l}+R_{i}}\right)^{2}=\frac{1}{2} R_{l}\left(\frac{K_{t} \dot{z}}{R_{l}+R_{i}}\right)^{2} .
$$

Substituting the maximum value for $\dot{z}$, which is $Z_{r} \omega_{n}$ in (8), the power supplied to the load is given by

$$
P_{l-\text { out }}=\frac{1}{2} \frac{R_{l}}{\left(R_{l}+R_{i}\right)^{2}} \omega_{n}^{2} K_{t}^{2} Z_{r}^{2} .
$$

Equation (9) shows the relationship between the relative displacement, excitation frequency, load resistance and the harvested power from a given generator.

Now, we design an optimum energy harvesting system to extract maximum energy from a given vibration source with known amplitude and frequency of oscillation. This design will be accomplished based on the parameters of a given generator that has given $K_{t}$ and $R_{i}$ values. It is also assumed that, due to the transducer size limits, the maximum displacement of the oscillating mass is specified. Therefore, the aim of design is the optimal selection of system parameters including $k, R_{l}$ and $m$ to harvest the maximum power from the given generator within the specified range of motion. To this end, considering $Z_{r_{0}}$ as the maximum allowable relative displacement of mass (i.e., $Z_{r}=Z_{r_{0}}$ is constant), the maximum value of (9) is obtained when the load resistance is equal to the internal resistance of the generator in which the output power is $\omega_{n}^{2} k_{t}^{2} Z_{r_{0}}^{2} / 8 R_{i}$. The mass can then be selected from (7) to limit its maximum displacement to $Z_{r}=Z_{r_{0}}$,

$$
m=\frac{Z_{r_{0}}}{Y \omega_{n}}\left(c_{m}+\frac{K_{t}^{2}}{2 R_{i}}\right) .
$$

The natural frequency of system is equal to the excitation frequency when $k=m \omega^{2}$, considering $\omega=\omega_{n}$ in this condition the spring stiffness is given by

$$
k=\omega_{n} \frac{Z_{r_{0}}}{Y}\left(c_{m}+\frac{K_{t}^{2}}{2 R_{i}}\right) .
$$

\subsection{Power Generation from a constrained rotational energy harvesting transducer}

A rotational energy harvesting system comprising a sprung mass coupled to an electrical generator through a motion transmission system. The Ball screw is a conventional mechanism that converts linear motion to the rotational one. A schematic diagram of this type of system is shown in figure 3 . In this device the base movement causes the mass to vibrate. The ball screw then converts the low frequency linear motion of the seismic mass to high speed rotation. The governing differential equation of motion, having an ideal ball screw, igure 3 is written as

$$
\left(m+J\left(\frac{2 \pi}{l}\right)^{2}\right) \ddot{z}+\left(\frac{2 \pi}{l}\right)^{2}\left(c_{e}+c_{b g}\right) \dot{z}+k z=-m \ddot{y} .
$$




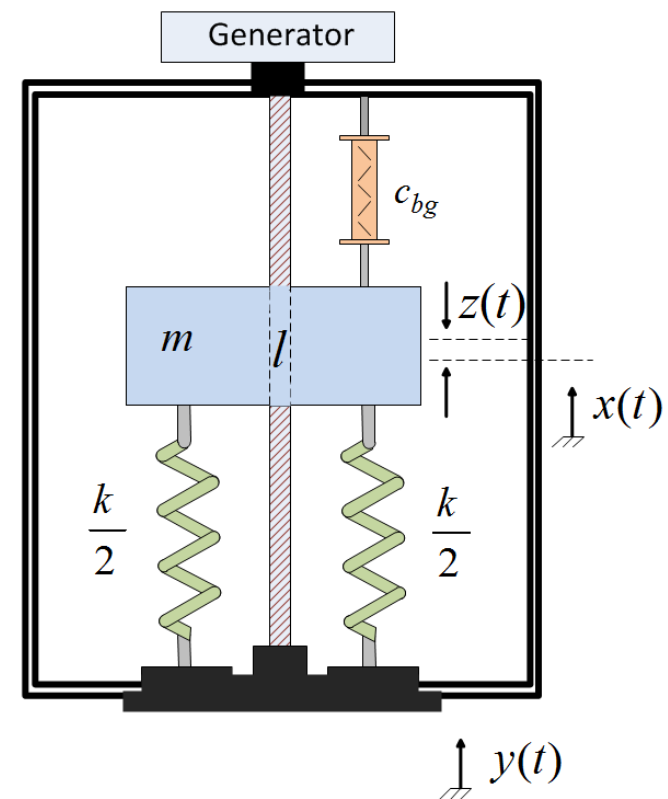

Figure 3. Schematic diagram of an energy harvesting system consisting of a sprung mass coupled to a generator through a ball screw

Considering $l$ as the ball screw lead, the equivalent reflected moment of inertia of the ball screw and the generator is given by $J(2 \pi / l)^{2}$, where $J$ refers to the total moment of inertia of the system including the moment inertia of the ball screw $J_{b}$ and generator $J_{g}$ and is defined as

$$
J=J_{g}+J_{b}
$$

Also $c_{b g}$ includes the mechanical viscous damping of the combined ball screw connections $c_{m b}$ and generator $c_{m g}$, i.e.,

$$
c_{b g}=c_{m b}+c_{m g} .
$$

For a harmonic base excitation $y=Y \sin \left(\omega t+\varphi_{y}\right)$, the amplitude of the relative displacement is

$$
\left|\frac{Z}{Y}\right|=\frac{m \omega^{2}}{\left[\left(k-\left(m+J\left(\frac{2 \pi}{l}\right)^{2}\right) \omega^{2}\right)^{2}+\left(\left(\frac{2 \pi}{l}\right)^{2}\left(c_{e}+c_{b g}\right) \omega\right)^{2}\right]^{1 / 2}} .
$$

The systems operates at its natural frequency when $\omega=\omega_{n}$ where $\omega_{n}$ is given by

$$
\omega_{n}=\left[\frac{k}{m+J\left(\frac{2 \pi}{l}\right)^{2}}\right]^{1 / 2}
$$

In this condition, the relative displacement of the mass is given by

$$
Z_{r}=\frac{m Y \omega_{n}}{\left(\frac{2 \pi}{l}\right)^{2}\left(c_{b g}+\frac{T_{i}^{2}}{R_{i}+R_{l}}\right)},
$$

where $T_{i}$ is the rotary generator emf-constant which is equivalent to $K_{t}$ in linear systems. Then the output power is therefore expressed as 


$$
P_{b-\text { out }}=\frac{1}{2} \frac{R_{l}}{\left(R_{l}+R_{i}\right)^{2}} T_{i}^{2}\left(\frac{2 \pi}{l}\right)^{2} \omega^{2} Z_{r}^{2} .
$$

Now, we assume that we wish to determine the parameters of a rotational system (i.e., $l, m$ and $R_{l}$ ), based on a specific generator and given mass (i.e., known $T_{i}, R_{i}$ and $m$ ), such that the maximum energy can be extracted from a specified vibration source (i.e., known $Y$ and $\omega$ ).

Considering the maximum allowable displacement of mass $Z_{r}=Z_{r_{0}},(2 \pi / l)^{2}$ is then obtained from (17) as

$$
\left(\frac{2 \pi}{l}\right)^{2}=\frac{m Y \omega_{n}}{Z_{r_{0}}\left(c_{b g}+\frac{T_{i}^{2}}{R_{i}+R_{l}}\right)} .
$$

By replacing (19) in (18), the output power for the constrained system is obtained from the following equation

$$
P_{b-\text { out }}=\frac{1}{2} \frac{R_{l}}{\left(R_{l}+R_{i}\right)^{2}} T_{i}^{2} \frac{m Y \omega_{n}}{Z_{r_{0}}\left(c_{b g}+\frac{T_{i}^{2}}{R_{i}+R_{l}}\right)} \omega_{n}^{2} Z_{r_{0}}^{2} .
$$

From (20), the optimum load resistance to harvest the maximum power is obtained from $\partial P_{b-o u t} / \partial R_{l}=0$, which results in

$$
R_{l, \text { rotational }, P_{\max }}=\left(R_{i}^{2}+R_{i} \frac{T_{i}^{2}}{c_{b g}}\right)^{1 / 2},
$$

the optimum ball screw lead can be derived as

$$
l=2 \pi\left[\frac{Z_{r_{0}}}{m Y \omega_{n}}\left(c_{b g}+\frac{T_{i}^{2}}{R_{i}+\left(R_{i}^{2}+R_{i} \frac{T_{i}^{2}}{c_{b g}}\right)^{1 / 2}}\right)\right]^{1 / 2},
$$

and the spring stiffness can be calculated from (16).

\section{Power and efficiency comparison between linear and rotational systems}

\subsection{Power and efficiency of an electromagnetic constrained transducer}

Efficiency is a fundamental term that has been studied for different energy harvesting systems. Relying solely on the assessment of the output power of energy harvesters does not reflect their quality of performance and their capability to harvest the maximum amount of power. However, in the context of vibration harvesting systems, the concept of efficiency has received less attention in the literature than that of maximizing output power. Traditionally, efficiency is defined as the ratio of the electrical power output to the mechanical input power; whilst, in a vibration-based energy harvester, the input mechanical power itself is related to the device characteristics. Also, the efficiency cannot be defined in terms of the potential mechanical power available from the source as, in some applications, the loading by the harvester does not influence the dynamics of the source of vibration. Hence, the potential mechanical power available from the source is effectively limitless [14]. To compare the power output of various transducers, a dimensionless figure of merit, called effectiveness $e$, is introduced by Roundy [28] which is defined as

$$
e=\kappa^{2} Q^{2} \frac{\rho}{\rho_{0}} \frac{\lambda}{\lambda_{\max }}
$$


where, $Q$ is the quality factor and is related to the damping ratio of the system, $\kappa$ is the coupling coefficient of the transduction mechanism, $\rho$ is the actual density of the device, $\rho_{0}$ is a baseline density, $\lambda$ is the actual transmission coefficient and $\lambda_{\max }$ is the maximum transmission coefficient. However, in the "effectiveness" index, $Q$ is related to the damping ratio of the system and does not have a fundamental limit. Hence, this metric comparison does not reveal how well the device is optimized [8]. To investigate how close a device is to its optimum performance and distinguish between different proof mass densities and geometries, Mitcheson et al. [14] introduce a "volume figure of merit", defined as

$$
\text { FoM }_{V}=\frac{P_{\text {out }}}{\frac{1}{16} Y_{0} \rho_{A u} V_{0}^{\frac{4}{3}} \omega^{3}} .
$$

This dimensionless ratio compares the performance of the device with that of an ideal device. The device has the same total package volume but with a proof mass equal to the density of gold ( $\rho_{A u}$ ), occupying half of this volume $\left(V_{0}\right)$. The proof mass oscillates in the other half of this package. The power output harvested by this hypothetical device is considered as the maximum possible output for the based vibration with amplitude of $Y_{0}$ at frequency of $\omega$. The power output of the transducer is compared with the maximum possible output to evaluate the performance of a device as a function of its overall size. Although the "volume figure of merit" facilitates the comparison of a harvesting device performance with a reference ideal energy harvesting system, it does not enable the calculation of input power absorbed by the system to produce a certain amount of output power.

Elliott and Zilletti [27] conducted research into scaling of linear electromagnetic transducers for power harvesting and shunt damping. In this study the efficiency is defined as the ratio of output power to the sum of the mechanical dissipated power, electrical power loss and electrical output power. This definition is closer to the original definition of efficiency. This study shows that the efficiency of a linear electromagnetic transducer depends on a non-dimensional electromechanical coupling coefficient which will be discussed later in this paper. The coupling coefficient scales with the transducer's size. However, this research does not consider the constraint on the displacement of the proof mass. The mechanical input power absorbed by the energy harvesting structure is given by

$$
P_{l-i n}=\frac{1}{2}\left(c_{m}+\frac{K_{t}^{2}}{R_{l}+R_{i}}\right) \omega_{n}^{2} Z_{r}^{2} .
$$

Here, we define the efficiency of a linear system, $E_{l}$, as the ratio of the electrical power harvested from (9) to the supplied mechanical power from (25), which is

$$
E_{l}=\frac{P_{l-o u t}}{P_{l-i n}}=\frac{R_{l} K_{t}^{2}}{c_{m}\left(R_{l}+R_{i}\right)^{2}+K_{t}^{2}\left(R_{l}+R_{i}\right)} .
$$

The load resistance corresponding to the maximum efficiency of the system, as opposed to the maximum power output, can be obtained from $\partial E_{l} / \partial R_{l}=0$, i.e,. differentiation of (26), which results in

$$
R_{l, \text { linear }, E_{\max }}=\left(R_{i}^{2}+R_{i} \frac{K_{t}^{2}}{c_{m}}\right)^{1 / 2} .
$$

By comparing the optimum load resistance for maximum output power $\left(R_{l, \text { linear }, P_{\max }}=R_{i}\right)$, and the load resistance corresponding to the maximum achievable system efficiency derived in (27), it is realized that the latter is always greater than the former. Therefore, in a practical linear system it is not possible to achieve maximum efficiency at the maximum output power point. The mechanical input power absorbed by the rotational system can be calculated as

$$
P_{b-i n}=\frac{1}{2}\left(c_{b g}+\frac{T_{i}^{2}}{R_{i}+R_{l}}\right)\left(\frac{2 \pi}{l}\right)^{2} \omega_{n}^{2} Z_{r}^{2} .
$$


The harvesting efficiency, $E_{b}$, is defined as

$$
E_{b}=\frac{P_{b-o u t}}{P_{b-i n}}=\frac{R_{l} T_{i}^{2}}{\left(R_{i}+R_{l}\right)^{2} c_{b g}+T_{i}^{2}\left(R_{i}+R_{l}\right)} .
$$

Also, from (29), the load resistance corresponding to the maximum efficiency of the system can be obtained from $\partial E_{b} / \partial R_{l}=0$, which is

$$
R_{l, \text { rotational }, E_{\max }}=\left(R_{i}^{2}+\frac{R_{i} T_{i}^{2}}{c_{b g}}\right)^{1 / 2} .
$$

Comparison of (21) and (30) reveals that in the rotational system, the optimum load resistance to obtain the maximum efficiency is the same as the load resistance corresponding to the maximum power. In the other words, for a constrained rotational system the maximum efficiency occurs at the maximum output power.

\subsection{Comparison of output power and efficiency of systems}

By replacing (7) in (9) for $Z_{r}=Z_{r_{0}}$ the load power of a constrained linear energy harvesting system for the load resistance corresponding to the maximum output power $\left(R_{l, \text { linear }, P_{\max }}=R_{i}\right)$, is

$$
P_{l-\text { out }, P_{\max }}=\frac{\Lambda_{e m}}{8\left(1+\frac{\Lambda_{e m}}{2}\right)} m \omega_{n}^{3} Y Z_{r_{0}},
$$

where $\Lambda_{e m}$ is a non-dimensional electromechanical coupling coefficient of an energy harvesting system and is defined as [27]

$$
\Lambda_{e m}=\frac{K_{t}^{2}}{c_{m} R_{i}}
$$

for linear systems and

$$
\Lambda_{e m}=\frac{T_{i}^{2}}{c_{b g} R_{i}},
$$

for rotational systems. By increasing this coefficient (i.e., $\Lambda_{e m} \rightarrow \infty$ ) the maximum output power, given by (31), approaches the following expression

$$
\lim _{\Lambda_{e m} \rightarrow \infty} P_{l-o u t, P_{\max }}=\frac{1}{4} m \omega_{n}^{3} Y Z_{r_{0}} .
$$

This shows that the maximum theoretical power is determined by the environmental vibration characteristics $\left(\omega_{n}, Y\right)$ and also the system mass and the maximum allowable displacement. Note that $\omega_{n}$ is a characteristic of the transducer, but here the system is designed such that the undamped natural frequency of the device is equal to the frequency of excitation.

Considering (26), the efficiency of a constrained linear system for the load resistance corresponding to the maximum output power $\left(R_{l, \text { linear }, P_{\max }}=R_{i}\right)$, can readily be shown to be [27]

$$
E_{l, P_{\max }}=\frac{\Lambda_{e m}}{4+2 \Lambda_{e m}} .
$$

For weak linear coupled systems, the efficiency is low. By increasing $\Lambda_{e m}$ the efficiency increases until it reaches a maximum value of $50 \%$, i.e.

$$
\lim _{\Lambda_{e m} \rightarrow \infty} E_{l, P_{\max }}=\frac{1}{2} .
$$


However, considering the optimum load resistance for rotational systems from (21), the output power of such systems from (20) can be written as

$$
P_{b-\text { out }, P_{\max }}=\frac{1}{2} \frac{\Lambda_{e m}\left(\sqrt{1+\Lambda_{e m}}\right)}{\left(1+\sqrt{1+\Lambda_{e m}}\right)^{2}+\Lambda_{e m}\left(1+\sqrt{1+\Lambda_{e m}}\right)} m \omega_{n}^{3} Y Z_{r_{0}},
$$

and for the case when $\Lambda_{e m} \rightarrow \infty$, the power is

$$
\lim _{\Lambda_{e m} \rightarrow \infty} P_{b-o u t, P_{\max }}=\frac{1}{2} m \omega_{n}^{3} Y Z_{r_{0}} .
$$

Also, the efficiency of rotational systems corresponding to the maximum output power can be obtained by replacing (30) in (28) and using (22), (32-a) and (32-b), which results in

$$
E_{b, P_{\max }}=\frac{\Lambda_{e m}\left(\sqrt{1+\Lambda_{e m}}\right)}{\left(1+\sqrt{1+\Lambda_{e m}}\right)^{2}+\Lambda_{e m}\left(1+\sqrt{1+\Lambda_{e m}}\right)},
$$

Equation (38) indicates that in the case of a rotational system, it is possible to achieve an efficiency of more than 50\%. To achieve such favourable design, the condition below must be met

$$
2\left(1+\sqrt{1+\Lambda_{e m}}\right) \leq \Lambda_{e m}
$$

This condition is satisfied if $\Lambda_{e m} \geq 8$. Selecting the parameters according to this condition can lead to a system with an efficiency above $50 \%$. For the case when $\Lambda_{e m} \rightarrow \infty$, the efficiency of the rotational system is

$$
\lim _{\Lambda_{e m} \rightarrow \infty} E_{b, P_{\max }}=1
$$

In the case that the a linear and a rotational system have same seismic mass, by replacing (7) in (25) and (17) in (28), for $Z_{r}=Z_{r_{0}}$, it an be shown that the mechanical input power for both systems is $1 / 2 m \omega_{n}^{3} Y Z_{r_{0}}$, however, the linear system in the optimum condition can only transfer less than half of this power to the load, while, the rotational system under certain condition, i.e. $\Lambda_{e m} \geq 8$, can harvest more power.

\subsection{Effect of the Scaling of constrained electromagnetic harvesters on the output power and efficiency}

It was shown earlier that by increasing $\Lambda_{e m}$, the efficiency of a typical energy harvesting transducer is improved. A question that arises here is "how do the output power and efficiency of a system change by increasing the size of the generator?".

Elliott and Zilletti [27] studied the relation between $\Lambda_{e m}$ and the characteristic length of a transducer $[L]$. In this study, assuming that $A_{w}$ is the cross-sectional area of the wire used for the coil of the electromagnetic transducer and $\mu_{w}$ is its resistivity, the resistance of the coil is given by

$$
R_{i}=\mu_{w} \frac{h}{A_{w}} .
$$

Here $h$ is the coil's wire length, which is approximately given by

$$
h=\frac{V_{C}}{A_{w}},
$$


where $V_{C}$ is the volume of the coil. For a well-designed transducer with saturated magnetic flux density $B$, the emf-constant ( $K_{t}$ for linear systems and $T_{i}$ for rotational systems) is proportional to the magnetic flux density times the length of the wire in the coil (i.e., $K_{t}$ or $T_{i}=B h$ ). Therefore, the electromechanical coefficient of the transducer can be re-written as

$$
\Lambda_{e m}=\frac{(B h)^{2}}{\mu_{w} \frac{h}{A_{w}} c_{m}}=\frac{B^{2} V_{C}}{\mu_{w} c_{m}} .
$$

The magnetic flux density $(B)$ and wire resistivity $\left(\mu_{w}\right)$ of the transducer depend on their material properties, but not on the transducer dimensions. In general, the scale of the volume of the coil $\left(V_{C}\right)$ is $\left[L^{3}\right.$ ], whereas the mechanical damping coefficient ( $c_{m}$ for linear systems and $c_{b g}$ for rotational systems) is related to the structure and the detailed mechanism of the transducer, but generally scales as $[L][29]$. Therefore, the electromechanical coefficient shown in (32-a) and (32-b) is proportional to the square of the characteristic length of the transducer $\left[L^{2}\right]$. Hence, an option in increasing the coupling coefficient of a transducer is to increase its overall size. From (33) and (37) it is evident that, for both systems, by increasing the size of device the electromagnetic coefficient and consequently the output power of the system is increased.

In the case of a rotational system, considering the combined ball screw, mass, spring and the rotary generator as the transducer assembly, the coupling coefficient related to the generator part of the transducer can be defined as

$$
\Lambda_{\text {emg }}=\frac{T_{i}^{2}}{c_{m g} R_{i}},
$$

where $c_{m g}$ is the mechanical damping associated with the rotary generator. According to the discussion presented above, it is expected that $\Lambda_{\text {emg }}$ will scale with the square of the characteristic length of the generator $\left[L^{2}\right]$. This assumption will be examined in the next section by studying the specifications of a set of commercial generators. For the rotational transducer assembly, the coupling coefficient defined in (32-b) can be rewritten as

$$
\Lambda_{e m}=\frac{T_{i}^{2}}{\left(c_{m g}+c_{m b}\right) R_{i}},
$$

where $c_{m b}$ is the mechanical damping due to the presence of other transducer's mechanical components such as ball screw, bearings and coupling shafts. Here, by increasing the size of the rotary generator, the quantity $T_{i}^{2} / R_{i}$ scales as $\left[L^{3}\right]$, but $c_{m g}$ scales as $[L]$, while, $c_{m b}$ does not scale up. Hence, it can be understood that by increasing the generator size, the coupling coefficient of the overall transducer assembly is increased but due to constant $c_{m b}$, the rate of scaling is higher than $\left[L^{2}\right]$. For instance if two rotational systems are designed based on two different rotary generators with electromechanical coefficients $\Lambda_{e m g_{1}}$ and $\Lambda_{e m g_{2}}$, the ratio of the non-dimensional electromechanical coefficient for these generators scales as $\left[L^{2}\right]$, i.e.,

$$
\frac{\Lambda_{e m g_{2}}}{\Lambda_{e m g_{1}}}=\frac{\frac{T_{i_{2}}^{2}}{c_{m g_{2}} R_{i_{2}}}}{\frac{T_{i_{1}}^{2}}{c_{m g_{1}} R_{i_{1}}}} \propto\left[L^{2}\right],
$$

and from (45) the ratio of the overall electromechanical coefficient of the designed transducers is 


$$
\frac{\Lambda_{e m_{2}}}{\Lambda_{e m_{1}}}=\frac{\frac{T_{i_{2}}^{2}}{\left(c_{m g_{2}}+c_{m b}\right) R_{i_{2}}}}{\frac{T_{i_{1}}^{2}}{\left(c_{m g_{1}}+c_{m b}\right) R_{i_{1}}}}=\frac{\Lambda_{e m g_{2}}}{\Lambda_{e m g_{1}}} \times \frac{1+\frac{c_{m b}}{c_{m g_{1}}}}{1+\frac{c_{m b}}{c_{m g_{2}}}} .
$$

Therefore, if $c_{m b} / c_{m g_{1}}$ is greater than $c_{m b} / c_{m g_{2}}$, then in comparison with $\Lambda_{e m_{1}}, \Lambda_{e m_{2}}$ scales with a ratio greater than $\left[L^{2}\right]$.

\section{Numerical study}

\subsection{Linear system examples}

This section investigates the relation between size and efficiency of energy harvesting devices under constrained condition brought about by the employed commercial generator. It is assumed that a source of vibration (for example a vertical movement of a boat) with a frequency of $0.5 \mathrm{~Hz}(\omega=\pi \mathrm{rad} / \mathrm{sec}$ ) and amplitude of $1 \mathrm{~m}(Y=1 \mathrm{~m})$ is available. We are required to design an energy harvesting device such that the maximum displacement of the seismic mass does not exceed $0.3 \mathrm{~m}$.

First case is dedicated to the design of a linear energy harvesting structure based on figure 1. Table 1 lists the parameters of a variety of linear electromagnetic actuators presented in [27] that are sorted in the order of small to large scales. The last system represents a hypothetical case in which the size of the actuator is much larger than model ASP400 ( $~ 8$ times).

Table 1 Parameters of a number of linear electromagnetic inertial actuator models[27]

\begin{tabular}{lllllll}
\hline & $K_{t}$ & $R_{i}$ & $c_{m}$ & $\Lambda_{e m}$ & $m$ & $P_{l-\text { out }, P_{\max }}$ \\
Type & $(\mathrm{N} / \mathrm{A})$ & $(\Omega)$ & $(\mathrm{Ns} / \mathrm{m})$ & & $(\mathrm{kg})$ & $(\mathrm{W})$ \\
\hline Trust headphone actuator & 0.74 & 8 & 0.38 & 0.18 & 0.03 & 0.007 \\
Micromega(IA-01) & 1.6 & 3.0 & 1.4 & 0.61 & 0.17 & 0.09 \\
Aura & 7 & 4.4 & 9 & 1.23 & 1.39 & 1.23 \\
Motran (IFX 30-100) & 10 & 1.6 & 44 & 1.42 & 7.18 & 6.93 \\
Micromega (ADD-45N) & 20 & 4 & 35 & 2.86 & 8.11 & 11.10 \\
ASP 400 & 21 & 1.6 & 30 & 9.19 & 16.02 & 30.60 \\
Hypothetical case & 42 & 0.8 & 60 & 36.75 & 111.01 & 224.8 \\
\hline
\end{tabular}

For each presented linear actuator type, the proof mass is calculated such that the oscillation at excitation frequency $(\omega=\pi \mathrm{rad} / \mathrm{sec})$ occurs within the given constraint (i.e., $\left.Z_{r_{0}}=0.3 \mathrm{~m}\right)$. For each inertial generator $\Lambda_{e m}$ and the seismic mass are calculated from (32-a) and (10), respectively. Then, at optimum load resistance ( $R_{l, \text { linear }, P_{\max }}=R_{i}$ ), the output power is obtained from (9). As table 1 shows, by increasing the transducer dimensions, $\Lambda_{e m}$ is increased and that is in agreement with the result presented in section 3. Also, by increasing the size of the linear actuator, the overall damping of the system gets larger, thus, requiring a bigger mass to reach the same displacement (i.e., $Z_{r_{0}}=0.3$ ). In addition, it is seen that by increasing the size of the linear actuator, the output power increases. However, as in this case, mass is the design variable (and for hence the absorbed mechanical power is different for each design), system efficiency would therefore be a more appropriate criterion to be used in order to compare the different harvesters. Figure 4 shows the efficiency of the designed system corresponding to their maximum output power calculated from (26). It is seen that by increasing $\Lambda_{e m}$ due to the increase of the transducer size, the 
efficiency of the energy conversion system is improved. However, even in the case of a hypothetical system where the size has been increased dramatically, the efficiency of the system does not exceed 50\% which is in agreement with the result obtained from (35).

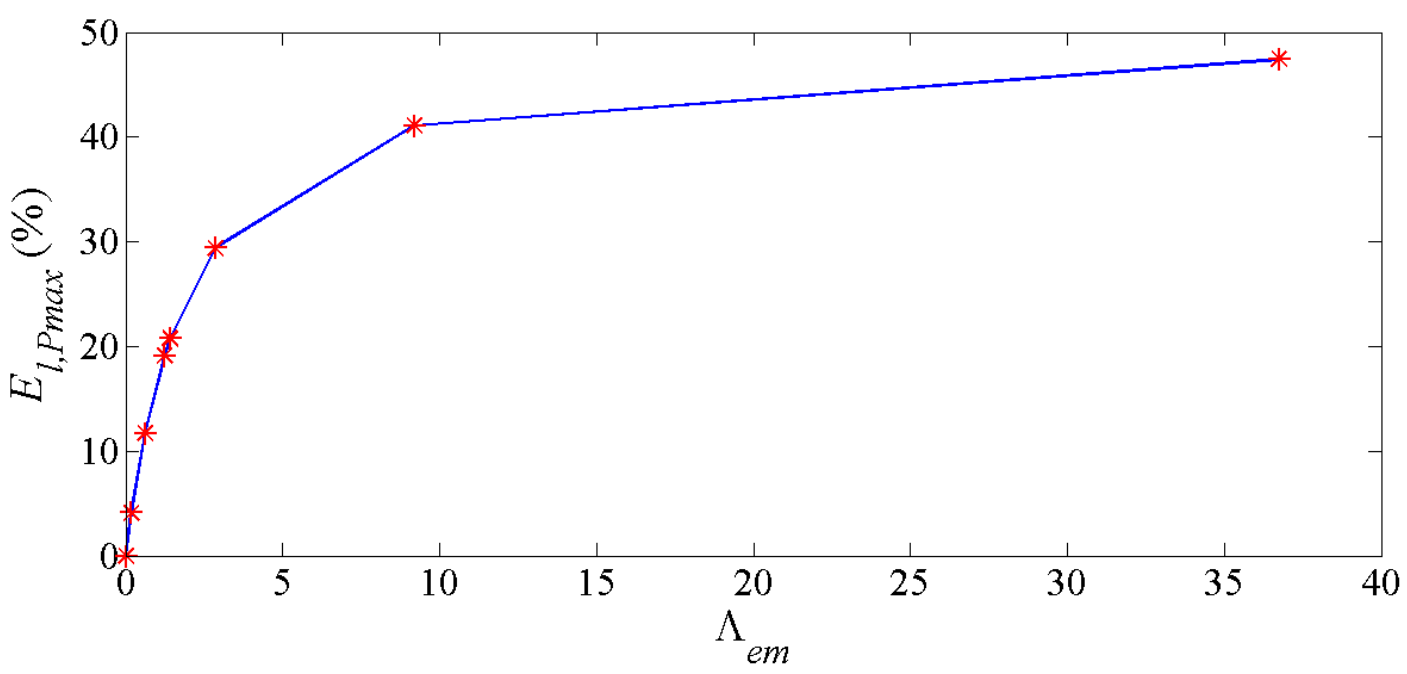

Figure 4. Efficiency of linear electromagnetic energy harvesting systems versus $\Lambda_{e m}$ for the linear actuator shown in table 1

\subsection{Rotational system examples}

Table 2 presents the size and specifications of a number of commercial PM (permanent magnet) generators where $h$ and $r$, respectively, are the length and the radius of the rotary generator coupled to the ball screw as presented in figure 3 . Here, for each generator, $\Lambda_{e m g}$ is calculated from (44), see table 2. Figure 5 shows the variation of the coupling coefficients of the generators in comparison with the size of the reference generator (Model a). A reasonable fit to $\Lambda_{\text {emg }}$ shows that it is linearly proportional to $\left(V_{i} / V_{1}\right)^{\frac{2}{3}}$, where $V_{1}$ is the volume of generator model a, and $V_{i}$ is the volume of the selected generator. This result validates the statement made in section 3 that the electromechanical coupling coefficient of a generator scales up with the square of the characteristic length of the device $\left[L^{2}\right]$. Also in each case $\Lambda_{e m}$ which represents the electromechanical coefficient of the transducer assembly is calculated from (45). Note that $c_{m b}$ is not a function of the generator size and is assumed to be $3.0 \mathrm{E}-3\left(\mathrm{mN} . \mathrm{m} . \mathrm{s} . \mathrm{rad}^{-1}\right)$ for all the designed transducers. A comparison of $\Lambda_{e m}$ and $\Lambda_{e m g}$ reveals that the $\Lambda_{e m}$ scales with a ratio higher than that of $\Lambda_{e m g}$. This agrees with the discussion presented in section 3 . 
Table 2. The parameters of PM motors from Faulhaber [30]

\begin{tabular}{llllllllll}
\hline & $h$ & $r$ & $T_{i}$ & $R_{i}$ & $c_{m g}$ & $\Lambda_{e m g}$ & $\Lambda_{e m}$ & \multicolumn{2}{l}{$P_{b-\text { out }, P_{\max }}$} \\
Type & $(\mathrm{mm})$ & $(\mathrm{mm})$ & $(\mathrm{mNm} / \mathrm{A})$ & $(\Omega)$ & $\left(\mathrm{mN} \cdot \mathrm{m} \cdot{\left.\mathrm{s} \cdot \mathrm{rad}^{-1}\right)}\right.$ & & & $(\mathrm{mm})$ & $(\mathrm{W})$ \\
\hline $\mathrm{a}$ & 6 & 20 & 1.13 & 9.1 & $6 \mathrm{E}-5$ & 2.33 & 0.05 & 1.2 & 0.4 \\
$\mathrm{~b}$ & 12 & 26 & 2.77 & 2.3 & $4.2 \mathrm{E}-4$ & 6.78 & 0.95 & 1.5 & 6.2 \\
$\mathrm{c}$ & 16 & 28 & 3.86 & 4.3 & $4.8 \mathrm{E}-4$ & 7.22 & 0.99 & 1.6 & 6.4 \\
$\mathrm{~d}$ & 20 & 36 & 6.34 & 3.4 & $1.3 \mathrm{E}-3$ & 9.20 & 2.75 & 2 & 12.0 \\
$\mathrm{e}$ & 30 & 56 & 12.74 & 1.6 & $6 \mathrm{E}-3$ & 16.20 & 10.80 & 3.8 & 20.6 \\
$\mathrm{f}$ & 35 & 64 & 14.52 & 0.6 & $1.4 \mathrm{E}-2$ & 24.40 & 20.20 & 6.1 & 24.2 \\
$\mathrm{~g}$ & 44 & 90 & 23.83 & 0.23 & $6 \mathrm{E}-2$ & 39.94 & 38.4 & 13.5 & 27.3 \\
\hline
\end{tabular}

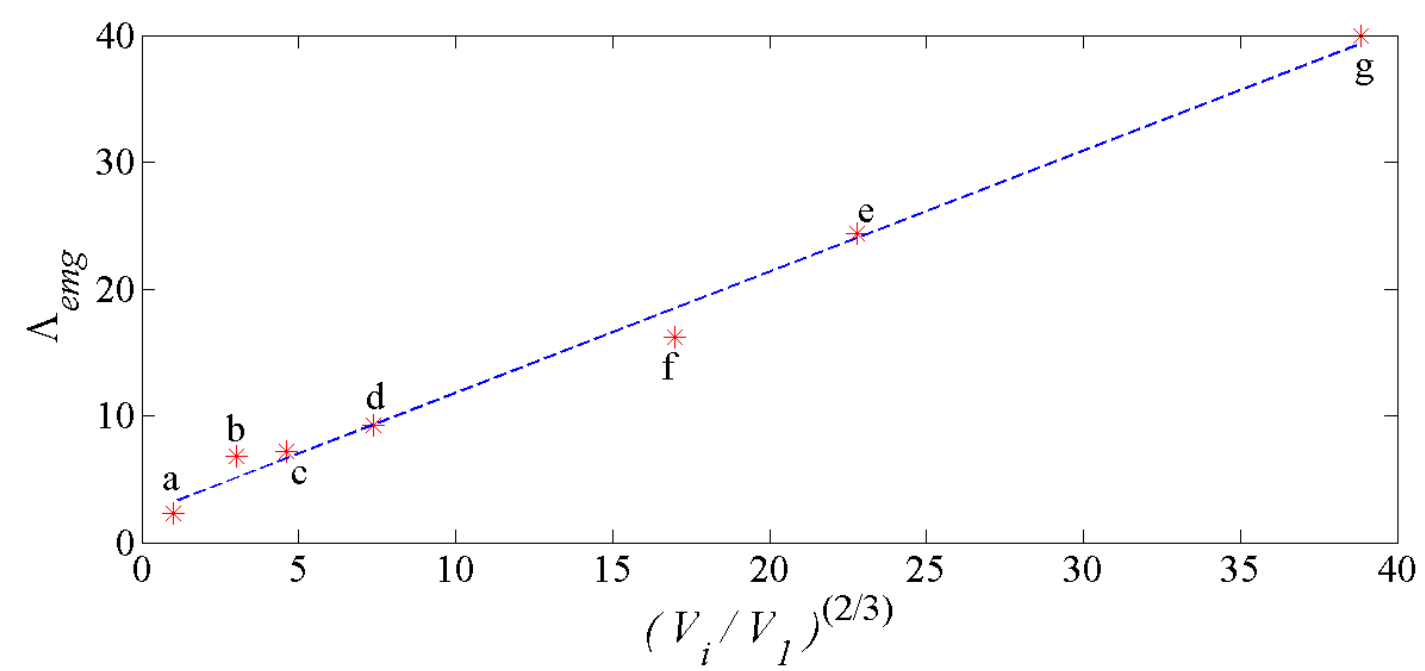

Figure 5. The coupling coefficient of rotary generators presented in table 2 versus ratio of their sizes to the reference generator in power of two over 3.

Now, it is assumed that the environmental vibration condition and the constraint on the maximum allowable displacement of the seismic mass are the same as the values considered in the first case $\left(Y_{0}=1 \mathrm{~m}, \omega=\pi\right)$. In this case, based on each of the PM generators presented in table 2, a rotational harvesting system is designed. It is assumed that the energy harvester has a mass of $8.1 \mathrm{~kg}$, and the design variables are $l$ and $R_{l}$. The optimum load resistance for each case is obtained from (21), and then the optimum lead size for the ball screw is calculated from (22). Table 2 presents the ball screw lead values and the generated power of each system corresponding to the relevant selected PM generator in each case. It is seen that by increasing the size of the generator, $\Lambda_{e m}$ and consequently the output power of the system is increased which is in agreement with (37).

Figure 6 shows the efficiency of the designed rotational systems versus $\Lambda_{e m}$. It is seen that by increasing the size of PM generators, the efficiency of the system increases. Here, in contrast with linear systems, an efficiency above $50 \%$ is achievable. This occurs for those systems whose $\Lambda_{e m}$ meet the criterion presented in (39), i.e., systems designed based on generators e, f and g. However, if $\Lambda_{e m}$ does not satisfy the condition presented in (39), i.e. $\Lambda_{e m} \geq 8$, designing a rotational energy harvesting system may result in a sub-optimum energy harvesting device in comparison with the linear system. For instance comparison of the designed systems based on the generators $\mathrm{a}, \mathrm{b}$ and $\mathrm{c}$ with the linear system designed based on Micromega (ADD-45N), reveals that although the rotational systems utilize the same mass, they produce less power compared with the linear system. Therefore, for constrained applications, in the 
design process of the energy harvesting systems, a rotary generator should be selected carefully to allow the designer to take advantage of the superiority of the rotational systems over the linear systems.

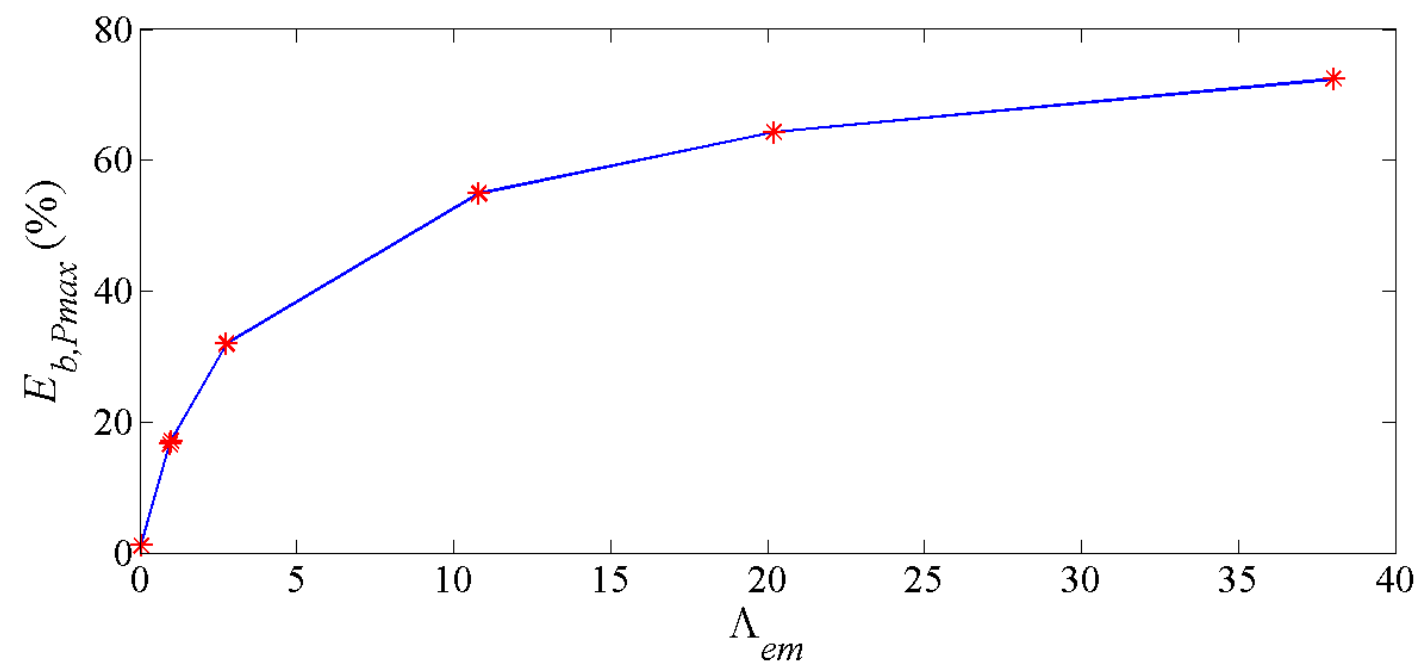

Figure 6. Efficiency of rotational electromagnetic energy harvesting systems versus $\Lambda_{e m}$ for the rotary generators shown in table 2

\section{Discussion and conclusion}

In some energy harvesting systems, the maximum displacement of the oscillating mass will be limited due to the physical constraints of the device. In systems where this limitation does not exist, choosing the optimum load resistance with the goal of maximizing the energy harvested from the environment is a process that takes place after the machine design. This is why, in these cases, the phrase "tuning" is used to refer to the selection of the resistance load. However, in systems where the maximum displacement of the mass is limited (constrained systems), choosing the optimum load resistance is part of the actual design process and cannot be done independently of choosing other parameters.

In this article, the maximum power condition and the corresponding efficiency for constrained vibration based linear and rotational energy harvesting devices were presented. For convenience, and for enabling the comparison of different systems, the definition for the coupling coefficient of an energy harvesting device given by (32) is employed [27].

In a linear system, electromechanical coupling coefficient $\left(\Lambda_{e m}\right)$ is shown to increase with the size of the transducer according to its characteristic length squared. However, in the case of a rotational system, although $\Lambda_{e m g}$ of the rotational generator, itself, increases as $\left[L^{2}\right]$, the value of $\Lambda_{e m}$ for the whole transducer assembly (including the ball screw) scales by a ratio greater than $\left[L^{2}\right]$.

It is shown that in a system with linear motion and constrained throw, even with the assumption of negligible mechanical losses, the maximum harvestable power (at optimum condition, i.e., $\left.R_{l, \text { linear }, P_{\max }}=R_{i}\right)$ is half of the mechanical power that can be absorbed by the transducer.

Also, it is shown that the output power and efficiency of linear systems increase by increasing the size of the structure. However, the maximum efficiency for such devices cannot be more than $50 \%$.

In contrast, rotational systems with a constrained throw show greater capability in transferring energy to the resistance load. In these systems, the ratio of the optimum load resistance and the internal resistance of the generator can be written according to equation (21) and (32) as follows:

$$
\frac{R_{l, \text { rotational }, P_{\max }}}{R_{i}}=\sqrt{1+\Lambda_{e m}} .
$$

Therefore, by increasing $\Lambda_{e m}$, this could be achieved by the enlargement of the rotary generator size, the ratio of the generator internal resistance to the load resistance increases. 
Figure 7 shows the logarithmic plot of $\Lambda_{e m}$ against the generator volumes over reference volume to the power of two over three for both linear and rotational transducers, respectively presented in tables I and II. The generators volume and the related reference volume for the linear transducers have been obtained from [27]. It is seen that $\Lambda_{e m}$ for rotational systems scales with a greater ratio in comparison with the linear systems. Hence, scaling the generator part in a rotational system, can be more beneficial in terms of improvement the system efficiency and output power.

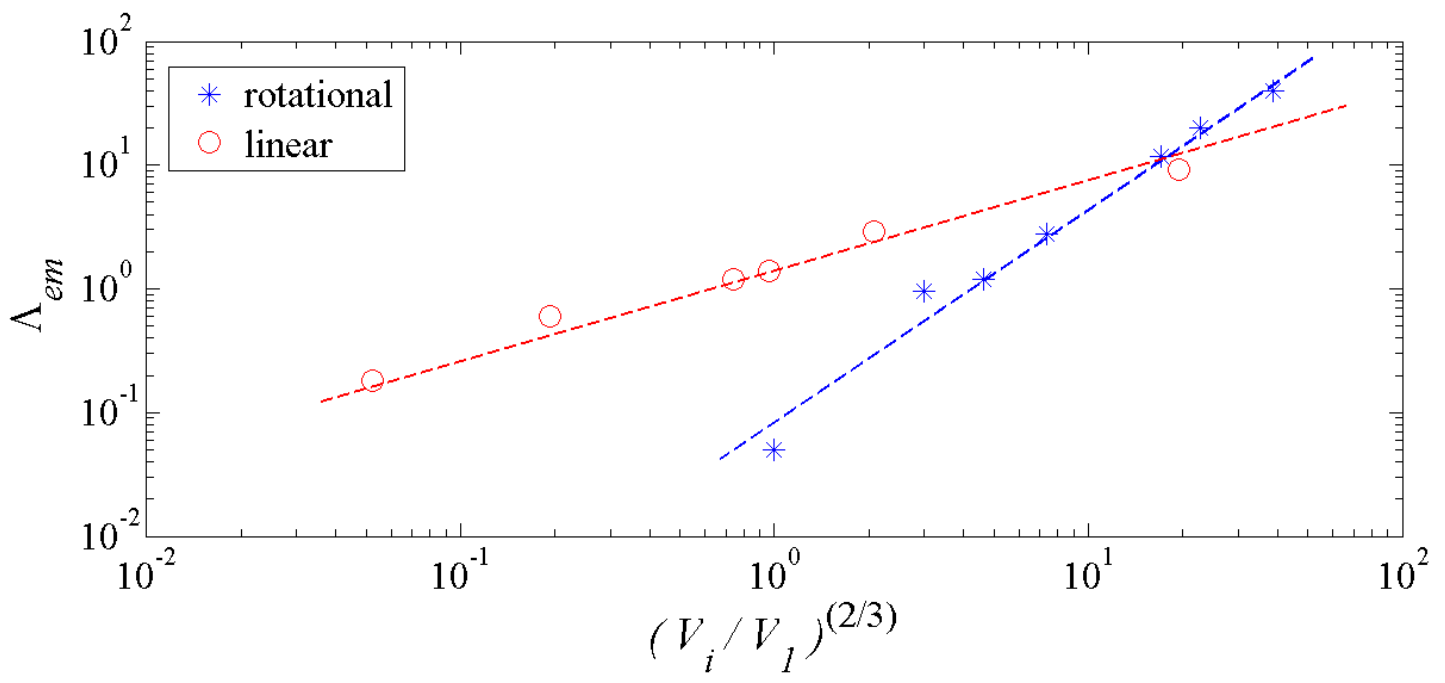

Figure 7. Log-log plot of $\Lambda_{e m}$ versus volume over the reference volume to the power of two over three for linear and rotational systems presented in tables I and II.

It is demonstrated that these transducers can be designed to operate with efficiencies above $50 \%$. The criterion that guarantees this superior efficiency was derived in (39) which can be used in the design process. This superiority of rotational systems over linear systems is due to the presence of an intermediate mechanism viz ball screw that can provide an extra design variable, thus enabling us to optimize the power output of the system subject to displacement constraint more desirably.

For a defined environmental condition and a given proof mass with constrained maximum allowable displacement, the amount of power delivered to the electrical load by a rotational system can be as high as twice the amount delivered by a linear system.

\section{Acknowledgements}

The authors are very grateful to Mr Mike Russell for providing financial support to the project.

\section{References}

1. Anton, S.R. and H.A. Sodano, A review of power harvesting using piezoelectric materials (20032006). Smart Materials and Structures, 2007. 16(3): p. R1-R21.

2. Khaligh, A., Z. Peng, and Z. Cong, Kinetic Energy Harvesting Using Piezoelectric and Electromagnetic Technologies\&\#x2014;State of the Art. Industrial Electronics, IEEE Transactions on, 2010. 57(3): p. 850-860.

3. Naruse, Y., et al., Electrostatic micro power generation from low-frequency vibration such as human motion. Journal of Micromechanics and Microenginering, 2009. 19: p. 1-5.

4. $\quad$ Bin, Y. and et al., Electromagnetic energy harvesting from vibrations of multiple frequencies. Journal of Micromechanics and Microengineering, 2009. 19(3): p. 035001.

5. Glynne-Jones, P., et al., An electromagnetic, vibration-powered generator for intelligent sensor systems. Sensors and Actuators A: Physical, 2004. 110(1-3): p. 344-349.

6. Beeby, S.P. and et al., A micro electromagnetic generator for vibration energy harvesting. Journal of Micromechanics and Microengineering, 2007. 17(7): p. 1257. 
7. Zhongjie, L., et al., Electromagnetic Energy-Harvesting Shock Absorbers: Design, Modeling, and Road Tests. Vehicular Technology, IEEE Transactions on, 2013. 62(3): p. 1065-1074.

8. Saha, C.R., et al., Optimization of an Electromagnetic Energy Harvesting Device. Magnetics, IEEE Transactions on, 2006. 42(10): p. 3509-3511.

9. Tang, X. and L. Zuo. Analysis of a micro-electric generator for microsystems. in IMECE. 2009. USA.

10. Rome, L.C., et al., Generating Electricity While Walking with Loads. Science, 2005. 309: p. 1725-1728.

11. Gupta, A., et al., Design of electromagnetic shock absorbers. International Journal of Mechanics and Materials in Design, 2006. 3(2): p. 285-291.

12. Sharkh, S.M., et al. An inertial coupled marine power generator for small boats. in Clean Electrical Power (ICCEP), 2011 International Conference on. 2011.

13. Renaud, M., et al., Optimum power and efficiency of piezoelectric vibration energy harvesters with sinusoidal and random vibrations. JOURNAL OF MICROMECHANICS AND MICROENGINEERING, 2012. 22(10): p. 105030.

14. Mitcheson, P.D., et al., Energy Harvesting From Human and Machine Motion for Wireless Electronic Devices. Proceedings of the IEEE, 2008. 96(9): p. 1457-1486.

15. Shu, Y.C. and I.C. Lien, Efficiency of energy conversion for a piezoelectric power harvesting system. JOURNAL OF MICROMECHANICS AND MICROENGINEERING, 2006. 16(11): p. 2429.

16. Stephen, N.G., On energy harvesting from ambient vibration. Journal of Sound and Vibration, 2006. 293(1-2): p. 409-425.

17. Cammarano, A., et al., Tuning a resonant energy harvester using a generalized electrical load. Smart Materials and Structures, 2010. 19(5): p. 055003.

18. Mansour, M.O., M.H. Arafa, and S.M. Megahed, Resonator with magnetically adjustable natural frequency for vibration energy harvesting. Sensors and Actuators A: Physical, 2010. 163(1): $\mathrm{p}$. 297-303.

19. Nakano, K., Y. Suda, and S. Nakadai, Self-powered active vibration control using a single electric actuator. Journal of Sound and Vibration, 2003. 260(2): p. 213-235.

20. Gargov, N.P., A.F. Zobaa, and G.A. Taylor. Direct drive linear machine technologies for marine wave power generation. in Universities Power Engineering Conference (UPEC), 2012 47th International. 2012.

21. Waters, R., et al., Experimental results from sea trials of an offshore wave energy system. Applied Physics Letters, 2007. 90(3): p. 034105-034105-3.

22. Choi, S.-B., M.-S. Seong, and K.-S. Kim, Vibration control of an electrorheological fluid-based suspension system with an energy regenerative mechanism. Journal of Automobile Engineering 2009. 223(D): p. 459-469.

23. Agamloh, E.B., A.K. Wallace, and A. von Jouanne, A novel direct-drive ocean wave energy extraction concept with contact-less force transmission system. Renewable Energy, 2008. 33(3): p. 520-529.

24. Matsuoka, T., et al., A Study of Wave Energy Conversion Systems Using Ball Screws Comparison of Output Characteristics of the Fixed Type and the Floating Type, in International Offshore and Polar Engineering Conference2002: Kitakyushu, Japan. p. 581-585.

25. Brown, P., D. Hardisty, and T.C.A. Molteno. Wave-powered small-scale generation systems for ocean exploration. in OCEANS 2006 - Asia Pacific. 2006.

26. Cassidy, I.L., J.T. Scruggsa, and S. Behrensb, Design and Experimental Characterization of an Electromagnetic Transducer for Large-Scale Vibratory Energy Harvesting Applications. Journal of Intelligent Material Systems and Structures, 2011. 22: p. 2009-24.

27. Elliott, S.J. and M. Zilletti, Scaling of electromagnetic transducers for shunt damping and power harvesting (ID 896), in ISMA2012: Leuven, Belgium. p. 463-474.

28. Roundy, S., On the Effectiveness of Vibration-based Energy Harvesting. Journal of Intelligent Material Systems and Structures 2005. 16(10): p. 809-823.

29. Peirs, J., Design of micromechateronic systems: scales laws, technologies and medical applications 2001, Katholike Universiteit Lueven.: Sweden.

30. Faulhaber, Brushless DC-Servomotors catalog, 2012. 